\title{
Measuring Trace Level Constituents with SEM/SDD-EDS
}

\author{
Dale Newbury and Nicholas Ritchie
}

National Institute of Standards and Technology, Gaithersburg, MD 20899, USA

For comparing limits of detection by spectroscopic methods, Wittry published a general equation that neatly separates the key spectrometric parameter of peak-to-background $(\mathrm{P} / \mathrm{B})$ from the physical factors for characteristic signal generation and detection [1]:

$$
\mathrm{C}_{\mathrm{mmf}} \sim[1 /(\mathrm{P} / \mathrm{B})][1 / \mathrm{Q} \varepsilon \beta \tau]^{1 / 2}
$$

where $\mathrm{C}_{\mathrm{mmf}}$ is the minimum mass fraction, $\mathrm{Q}$ is the cross section for production of the characteristic signal (events/incident particle/atom/unit area), $\varepsilon$ is the efficiency of signal detection (events detected/events produced, which includes both the geometric efficiency of the detector and the event quantum efficiency), $\beta$ is the exciting source brightness (incident particles/unit area/unit time), and $\tau$ is the measurement time. Consider the detection of trace constituents with electron excited x-ray spectrometry. The term "trace" will be arbitrarily defined as concentrations below 0.01 mass fraction while "major" is defined as C >0.1 and "minor" between 0.01 and 0.1 . Because P/B scales with a unit exponent while the other factors scale with the square root, equation (1) reveals the great advantage of wavelength dispersive x-ray spectrometry (WDS) over energy dispersive spectrometry (EDS) with semiconductor detection. For a given element, the P/B for WDS is generally a factor of 10 to 20 greater than that for EDS due to the greater resolution of WDS, e.g., $\sim 8 \mathrm{eV}$ for WDS (LiF diffractor) vs. $129 \mathrm{eV}$ for Si(Li)-EDS at MnK $\alpha$. Less obvious but very important, the WDS diffraction process excludes high count rate matrix peaks so that detector deadtime arises exclusively from the analyte element and matrix background. WDS will always yield a lower $\mathrm{C}_{\mathrm{mmf}}$ when a particular element is being sought. $\mathrm{Si}(\mathrm{Li})$-EDS has not generally been considered useful for trace level constituents.

But what if the analytical problem is such that any trace element may be of interest? Now the energy dispersive character of the measurement becomes important since a wide range of photon energies must be inspected at every location analyzed. For any spectrometric measurement, lowering the limit of detection depends upon accumulating more counts, although the improvement only proceeds as the square root of counts. The remarkable advances in output count rate (OCR) that can be achieved with the new class of silicon drift detector (SDD) EDS suggests that a re-evaluation of detection strategies for trace constituents may be in order. A cluster of four SDD chips with ganged output can exceed $500 \mathrm{kHz}$ OCR at a resolution of $127.5 \mathrm{eV}$ and more than $1 \mathrm{MHz}$ OCR while retaining a useful resolution $(\sim 145 \mathrm{eV})$ [2]. An extremely high count spectrum integral ( $>10 \mathrm{M}$ counts) can be obtained in $\sim 20-200 \mathrm{~s}$ with SDD-EDS, enabling the analyst to achieve interesting trace detection levels over a wide photon energy range. An example of what is possible for trace constituent detection with SDD-EDS is illustrated in Figure 1. NIST research glass $\mathrm{K} 523$ is a $\mathrm{Si}-\mathrm{Pb}-\mathrm{O}$ matrix with several elements at trace levels, with the composition listed in the table embedded in Figure 1. The spectrum was recorded with the QUAD SDD operating with a time constant of 440 ns (giving a 
resolution of $127.5 \mathrm{eV}$ at $\mathrm{MnK} \alpha$ ), a deadtime of $12 \%$ (to minimize coincidence), and an output count rate of $145 \mathrm{kHz}$, yielding a spectrum integral $(0.2-20 \mathrm{keV})$ of 28.9 million counts in 200s. All of the trace elements added to the glass can be found in the spectrum, although there are significant interferences for TiK-BaL. The remaining trace constituents, $\mathrm{Mg}, \mathrm{P}, \mathrm{Cr}, \mathrm{Ni}, \mathrm{Ge}$, and Eu can be readily detected, and a $\mathrm{C}_{\mathrm{mmf}}$ can be calculated from the peak and background counts and the known synthetic concentrations:

$$
\mathrm{C}_{\mathrm{mmf}}=\left[3 \mathrm{~B}^{1 / 2} /(\mathrm{P}-\mathrm{B})\right] \mathrm{C}_{\mathrm{s}}
$$

where $C_{s}$ is the concentration in the standard [3]. The detection limits range from 170 ppm for P to $390 \mathrm{ppm}$ for Eu. These levels are at least an order of magnitude below what has been routinely achieved in the past with $\mathrm{Si}(\mathrm{Li})-\mathrm{EDS}$. The measurement of trace $\mathrm{P}$ between the $\mathrm{SiK}$ and $\mathrm{PbM}$ families is especially impressive.

Routine analysis at the trace level with SEM/SDD-EDS will require implementation of a robust protocol to ensure that detected elements are indeed associated with the analyzed location and not a result of pathological remote electron scattering or long range x-ray fluorescence. The problem of sum peaks in SDD-EDS spectra, which are increasingly encountered at high OCR, must also be carefully addressed. When the trace peak of interest is located close to a peak of a major constituent, the detection problem is further exacerbated by the increased variance contributed by the major peak.

\section{References}

1. Wittry, D.B., Eur. Cong. Elec. Micros., Leiden, 3 (1980) 14.

2. Newbury, D., et al., Microsc Microanal 13, Suppl 2 (2007) 1340CD.

3. Goldstein, J.I. et al., Scanning electron Microscopy and X-ray Microanalysis, $3^{\text {rd }}$ ed., (Springer, New York, 2003) 446.

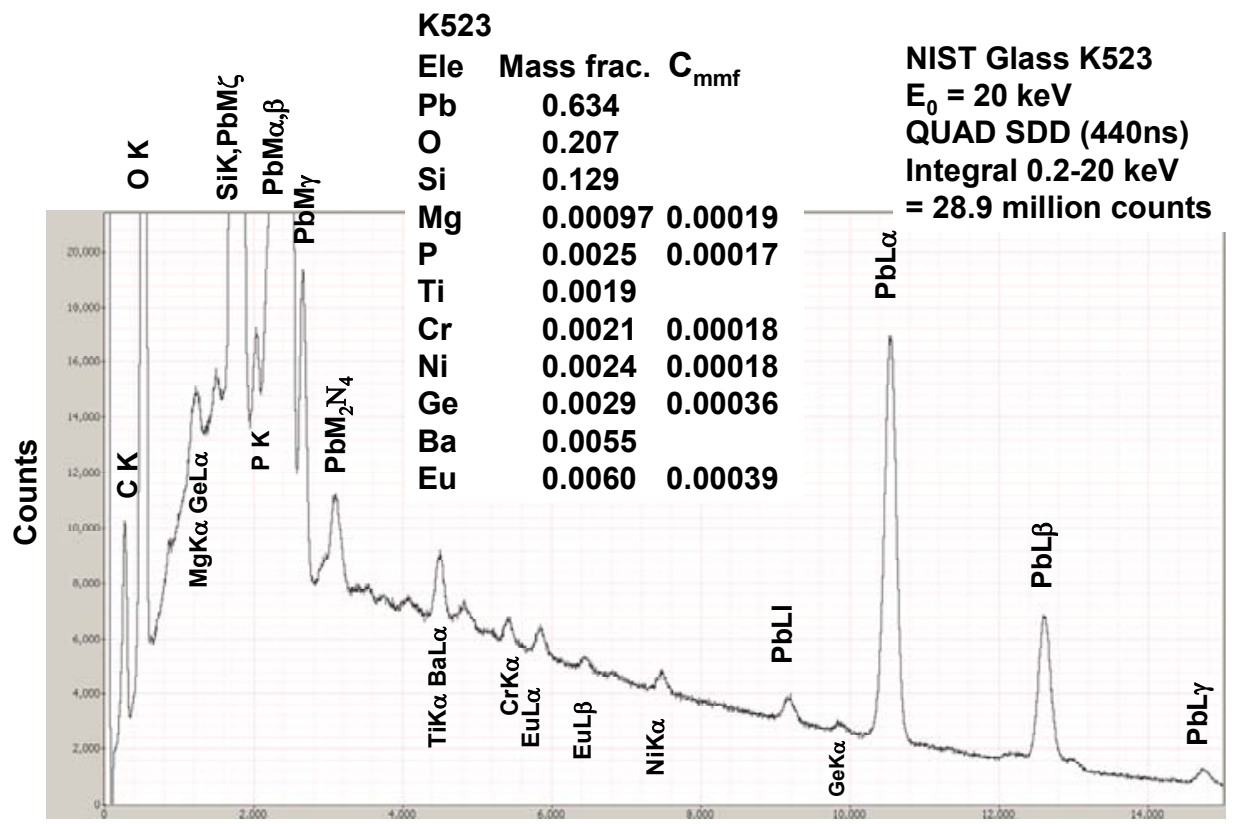

Photon Energy (eV)

Figure 1: SDD-EDS spectrum of NIST microanalysis research glass K523. 